\title{
Immunolocalization of glutaryl-CoA dehydrogenase (GCDH) in adult and embryonic rat brain and peripheral tissues
}

Olivier Braissant ${ }^{\mathrm{b}}$

Email : olivier.braissant@chuv.ch

Paris Jafari ${ }^{\mathrm{a}}$

Email : paris.jafari@chuv.ch

Noémie Remacle ${ }^{a}$

Email: noemie.remacle@chuv.ch

Hong-Phuc Cudré-Cung ${ }^{a}$

Email: thanh-hong-phuc.cung@chuv.ch

Sonia Do Vale Pereira ${ }^{a}$

Email: svalpereira@gmail.com

Diana Ballhausen ${ }^{a^{*}}$

* Corresponding author

Email: diana.ballhausen@chuv.ch

a Inborn Errors of Metabolism, Center for Molecular Diseases, Centre Hospitalier Universitaire Vaudois and University of Lausanne, 1011 Lausanne, Switzerland

${ }^{\mathrm{b}}$ Inborn Errors of Metabolism, Biomedicine, Centre Hospitalier Universitaire Vaudois and University of Lausanne, 1011 Lausanne, Switzerland 


\section{Abstract}

Glutaryl-CoA dehydrogenase (GCDH) is a mitochondrial enzyme that is involved in the degradation of tryptophan, lysine and hydroxylysine. Deficient enzyme activity leads to glutaric aciduria type-I (GA-I). This neurometabolic disease usually manifests with acute encephalopathic crises and striatal neuronal death in early childhood leading to an irreversible dystonic-dyskinetic movement disorder. Fronto-temporal atrophy and white matter changes are already present in the pre-symptomatic period. No detailed information on GCDH expression during embryonic development and in adulthood was available so far.

Using immunofluorescence microscopy and cell-type specific markers to localize GCDH in different tissues, we describe the differential cellular localization of GCDH in adult rat brain and peripheral organs as well as its spatiotemporal expression pattern. During embryonic development GCDH was predominantly expressed in neurons of the central and peripheral nervous system. Significant expression levels were found in epithelial cells (skin, intestinal and nasal mucosa) of rat embryos at different developmental stages. Besides the expected strong expression in liver, GCDH was found to be significantly expressed in neurons of different brain regions, renal proximal tubules, intestinal mucosa and peripheral nerves of adult rats.

GCDH was found widely expressed in embryonic and adult rat tissues. In rat embryos GCDH is predominantly expressed in brain implying an important role for brain development. Interestingly, GCDH was found to be significantly expressed in different other organs (e.g. kidney, gut) in adult rats probably explaining the evolving phenotype in GA-I patients.

\section{Contributions of authors}

DB got the research grant, initiated the project and determined the experimental design. $O B$ supervised the technical work and did most of data interpretation and creation of figures. PJ started the experimental work, but gave up by the end of her PhD thesis. The experimental work was completed by NR, SDVP and THPC. OB, PJ and $\mathrm{DB}$ wrote the manuscript. All authors participated in the revision of the manuscript and approved the final version. 


\section{Key words}

Glutaryl-CoA-Dehydrogenase;

expression;

glutaric aciduria type-l;

rat tissues;

embryonic development;

central and peripheral nervous system

\section{Animal Rights:}

All institutional and national guidelines for the care and use of laboratory animals were followed.

\section{Abbreviations:}

1 H-MRS: proton magnetic resonance spectroscopy, 3-OHGA: 3-hydroxy glutaric acid, BBB: blood brain barrier, BSA: bovine serum albumin, CNS: central nervous system, DEPC: diethyl-pyrocarbonate, GA: glutaric acid, GA-I: glutaric aciduria type I, GalC: galactocerebroside, GCDH: glutaryl-CoA dehydrogenase, GFAP: glial fibrillary acidic protein, KO: knockout, MAP-2: microtubule-associated protein 2, MBP: myelin basic protein, MRI: magnetic resonance imaging, MRPL12: mitochondrial ribosomal protein L12, NaDc3: sodium-dependent dicarboxylate transporter 3, NeuN: neuronal nuclei protein, OAT1: organic anion transporter, PBS: phosphate buffer saline, PFA: paraformaldehyde, $\mathrm{p}$-NFM: phosphorylated medium weight neurofilament

\section{Introduction}

Glutaryl-CoA dehydrogenase (EC 1.3.8.6; GCDH) is a nuclear encoded mitochondrial protein involved in the catabolic pathway of tryptophan, lysine and hydroxylysine. The functional enzyme is a homo-tetramer of $43.3 \mathrm{kDa}$ subunits that resides in the mitochondrial matrix (Lenich and Goodman, 1986b, a).

GCDH deficiency leads to glutaric aciduria type-I (GA-I, OMIM \# 231670). GA-I is an inborn error of amino acid metabolism belonging to the group of cerebral organic acidurias, which was until very recently thought to manifest exclusively with neurologic symptoms (Hoffmann et al., 1993). If untreated, GA-I presents with an 
acute encephalopathic crisis that usually occurs between the $6^{\text {th }}$ and $18^{\text {th }}$ month of life following a catabolic state due to gastroenteritis, febrile disease or immunization. During this crisis the child loses neurological functions following a severe neuronal damage typically in the caudate and putamen (Jafari et al., 2011a, b). Almost one third of identified patients under treatment still develop striatal injury leading to lifelong disability (Strauss and Morton, 2003). Even avoiding acute encephalopathic crisis, treated patients can show subtle but significant fine motor and speech deficits that witness chronic neurological damage (Beauchamp et al., 2009). Residual activity of GCDH (2-30\%) leads to low or undetectable excretion of GA and 3-OHGA in a subgroup of patients termed low excretors. In contrast, high excretors, with no residual GCDH activity, show the typical urinary metabolite pattern (Busquets et al., 2000).

Despite numerous in vitro and in vivo studies and generation of a $\mathrm{KO}$ mouse model for GA-I, the pathogenesis of this disease still remains poorly understood. We recently reviewed different hypotheses on mechanisms leading to brain damage in GA-I (Jafari et al., 2011b). Nevertheless, the only common opinion on GA-I neuropathology is the central role of accumulating metabolites glutaric acid (GA) and 3-hydroxy-glutaric acid (3OHGA) in the brain. 1H-MRS studies have recently shown that GA and 3-OHGA do not accumulate in basal ganglia, but in white matter of high excretor patients (Harting et al., 2015). These patients also showed decreased creatine and increased choline levels accompanied by white matter changes in MRI.

In mice, western blot and RT-PCR revealed a ubiquitous GCDH expression with highest levels in liver and kidney, consistent with its role in amino acid oxidation (Woontner et al., 2000). In brain, GCDH expression was described to be limited to neurons with the highest level in the cerebellum and lower levels in striatum and cortex (Woontner et al., 2000, Ballhausen et al., 2010). Post-mortem analysis of the distribution of GA and 3-OHGA in different body organs revealed the highest 3OHGA concentration in the putamen among all brain areas, which is in line with the pattern of neuronal damage in GA-I patients. However, these metabolites were also found largely in liver, kidney, heart and skeletal muscles, where they do not seem to induce toxic effects (Kolker et al., 2003). 
The hypothesis of de novo synthesis of GA and 3-OHGA in the brain (Sauer et al., 2006) and their intracerebral trapping due to limited efflux is supported by the 101000 times elevated metabolite concentrations found in the brain of affected patients (Kolker et al., 2004).

Little information is available on the localization of GCDH in adult tissues and its expression pattern during embryonic development. We analyzed the differential localization of GCDH in several adult tissues as well as during embryogenesis in the rat, a well-accepted in vivo model for numerous human brain pathologies.

\section{Experimental Procedures}

\section{Antibodies}

The following primary antibodies were used: rabbit polyclonal anti-GCDH (AV43559; Sigma-Aldrich, USA), mouse monoclonal anti-phosphorylated medium weight neurofilament ( $p$-NFM; Sigma-Aldrich, Israel), anti-glial fibrillary acidic protein antibody (GFAP; Millipore, USA), anti-galactocerebroside (GalC; Millipore, USA), anti-microtubule associated protein 2 (MAP2; Millipore, USA), and anti-neuronal nuclei antibody (NeuN; Millipore, USA), as well as goat polyclonal anti-myelin basic protein (MBP; Santa Cruz Biotechnology, USA). Secondary antibodies were: goat anti-rabbit IgG coupled to HRP (Biorad, USA), goat anti-rabbit IgG coupled to Alexa Fluor $^{\circledR} 555$ (Molecular Probes, USA), chicken anti-mouse IgG coupled to Alexa Fluor $^{\circledR} 448$ (Molecular Probes, USA), and donkey anti-Goat IgG coupled to Alexa Fluor $^{\circledR} 350$ (Molecular Probes, USA).

The specificity of the GCDH antibody was verified by western blotting, as described in details (Jafari et al., 2013). $45 \mu \mathrm{g}$ (brain) or $15 \mu \mathrm{g}$ (kidney, liver) of total proteins extracted from of wild type or $\mathrm{Gcdh}^{-1-}$ mice were loaded in each lane (see Figure 1A). For semi-quantitive determination of Gcdh expression in rat by western blotting, 30 $\mu \mathrm{g}$ of total protein for embryonic stages E15 and E18 as well as for adult tissues were loaded in each lane (see Figure 1B). MRPL12 was used as loading control.

\section{Preparation of tissues}

Adult female Sprague-Dawley rats purchased from Charles River, France were sacrificed by decapitation (handled according to the rules of the Swiss Academy for 
Medical Sciences) and their brain, liver, kidney, heart muscle, skeletal muscle, spleen and intestine were immediately dissected and rinsed in ice-cold DEPC-treated (Sigma, Switzerland) phosphate buffer saline (PBS). For the preparation of embryos, pregnant Sprague-Dawley rats at 13,15 and 17 days of gestation were sacrificed by decapitation and embryos were removed from the uterus and washed with ice-cold PBS. Organs and embryos were fixed in chilled $4 \%$ paraformaldehyde in PBS (PFAPBS; Sigma-Aldrich, Germany) overnight at $4^{\circ} \mathrm{C}$ followed by sequential infiltration with $12 \%$ and $18 \%$ sucrose (Calbiochem, Germany) in PBS at $4{ }^{\circ} \mathrm{C}$. Organs were then washed with PBS to remove the external excess of sucrose and embedded in O.C.T compound (Tissue-Tek, Netherland), frozen in liquid nitrogen-cooled isopentane (Sigma-Aldrich, Germany) and stored at $-80^{\circ} \mathrm{C}$ until used for cryosections.

\section{Immunofluorescence}

Cryosections $(12 \mu \mathrm{m})$ from pre-fixed tissues of at least three biological replicates were re-fixed for $15 \mathrm{~min}$ in $4 \%$ PFA-PBS at room temperature and washed in PBS (3x5 min). Permeabilization was performed with $0.1 \%$ Triton X-100 (Fluka, France) in PBS for $15 \mathrm{~min}$ followed by washes in PBS (4x5 min). Non-specific antibody binding sites were blocked for one hour at room temperature with 1\% BSA-PBS (SigmaAldrich, Germany). Primary antibody against GCDH was diluted 1:100 in 1\% BSAPBS and applied to sections overnight at $4^{\circ} \mathrm{C}$ in a moist chamber. After washing in PBS ( $3 \times 5 \mathrm{~min}$ ), sections were incubated for $1 \mathrm{~h}$ with the secondary antibody (dilution 1:100) directed towards rabbit-lgG and labeled with Alexa Fluor ${ }^{\circledR} 555$. Sections were then mounted under FluorSave ${ }^{\mathrm{TM}}$ reagent or processed to second primary antibody staining. Sections were further re-blocked for one hour at room temperature with $1 \%$ BSA-PBS and incubated overnight at $4^{\circ} \mathrm{C}$ with second primary antibodies directed towards MAP2, NeuN or p-NFM (all diluted 1:100) to reveal neurons, GFAP (1:100) to reveal astrocytes and MBP or GalC (1:100) to reveal oligodendrocytes. After washing in PBS ( $3 \times 5 \mathrm{~min})$, sections were incubated for $1 \mathrm{~h}$ with a secondary antibody directed towards mouse-lgG labeled with Alexa Fluor ${ }^{\circledR} 448$ (1:100) or goat-lgG labeled with Alexa Fluor ${ }^{\circledR} 350$ (1:100). Negative controls were processed the same but omitting the primary antibody, resulting in no staining. Sections were then mounted under FluorSave ${ }^{\mathrm{TM}}$, observed and digitized using an Olympus BX50 microscope equipped with a UC30 digital camera (Olympus, Japan). 


\section{Results}

\section{Specificity of the anti-GCDH antibody}

The specificity of the anti-GCDH antibody used in this study was ascertained by western blotting using tissues (brain, kidney and liver) extracted from wild type and $\mathrm{Gcdh}^{-/}$mice respectively. A single band at $45 \mathrm{kD}$ was specifically detected for GCDH in the three wild type tissues, while it could not be detected in tissues from $\mathrm{Gcdh}^{-1}$ mice (Figure 1; Gcdh/- mice kindly provided by Dr David Koeller, (Koeller et al., 2002)).

\section{GCDH expression in the adult rat}

The expression pattern of GCDH in adult rat tissues is presented in Figures 1B, 2 and 3 A-I, as well as summarized in Table 1. Semi-quantitative determination of Gcdh expression by western blotting revealed very high expression levels in liver, kidney and heart, moderate expression levels in brain and spleen and confirmed a significant expression already during embryonic development (Figure 1B).

\section{GCDH in the adult rat brain}

Co-staining with the different brain cell specific markers was performed in all brain regions. Only the most relevant results are shown in the respective figures. Parasagittal brain cryosections showed widespread GCDH expression throughout all brain areas (Figure $2 \mathrm{~A}-\mathrm{I}$ ), with the strongest levels observed in cerebral cortex (Figure 2 A,D-F) and Purkinje neurons of cerebellum (Figure 2 G-L). Basal ganglia (striatum; Figure 2 B), olfactory bulbs (Figure $2 \mathrm{C}$ ), hippocampus, thalamus, pons and medulla oblongata showed weak to moderate expression of the GCDH protein. Very interestingly, GCDH was found expressed in neurons (Figure 2 A,B,D-L) and oligodendrocytes (Figure $2 \mathrm{C}$; co-localization with MBP in olfactory bulbs) only, but could not be detected in astrocytes (data not shown). GCDH was expressed in the cell body of giant neurons (Figure 2 A,D-F: cortical pyramids, co-labeled with the neuronal markers NeuN and MAP-2 respectively ; and Figure 2 G-L: cerebellar Purkinje neurons co-labeled with the neuronal markers $p$-NFM and MAP-2 respectively). GCDH was regularly observed in both, neuronal dendrites (Figure 2 D$\mathbf{F}$ and J-L: cortical pyramidal dentritic trees and dendrites of cerebellar Purkinje neurons respectively, co-labeled with the dendritic marker MAP-2) and axons (Figure 
2 G-I: axons from basket cells and Purkinje neurons respectively, co-labeled with the axonal marker p-NFM).

\section{GCDH in adult peripheral tissues}

GCDH expression in adult peripheral tissues is presented in Figure 3 A-I and summarized in Table 1. As expected, a strong and homogenous GCDH expression was observed in liver parenchyma (Figure $3 \mathbf{A}$ ). While in kidney no GCDH immunoreactivity could be found in glomeruli, convoluted (most probably proximal) tubules of the renal cortex as well as of the juxtamedullary zone showed a strong GCDH expression (Figure 3 B,C). Sarcomers of skeletal muscles were strongly GCDH positive (Figure $3 \mathbf{D}$ ), while heart muscle showed a peculiar pattern of GCDH expression with certain cells staining positive while others not (Figure $3 \mathbf{E}$ ). Interestingly, intestinal epithelium showed a strong expression of GCDH in villi, including in the axon-like plexus of $L$ cells (Figure 3 F-I, including co-localization with the axonal marker p-NFM). Intestinal mucosa muscularis as well connective tissue were also positive for GCDH (Figure 3 F). Week GCDH expression could be observed in adult skin, while no immunoreactivity was detected in spleen (data not shown).

\section{GCDH expression in the rat embryo}

Tissue-specific differential GCDH expression in the rat embryo was analyzed the developmental stages E13, E15 and E17, during which the GCDH expression pattern did not vary much (Figures $3 \mathbf{J}-\mathbf{L}$ and 4 ; summarized in Table 1). High levels of GCDH protein were observed in the central and peripheral nervous systems. In particular, GCDH was strongly expressed in the cerebral cortex (Figure 4 A-C), basal ganglia (Figure $4 \mathbf{D}-\mathbf{F}$ ), cerebellum, as well as in the spinal cord neuroepithelium (Figure G-I). In CNS, GCDH was mainly localized to neurons, with a strong labeling in cell bodies and axons (Figure 4 D-F: co-localization in pallidum with the neuronal marker MAP-2; Figure 4 G-I: co-localization with the axonal marker-NFM, in particular in the spinal cord). GCDH was also strongly expressed in the peripheral nervous system, in particular in dorsal root ganglia and peripheral axonal bundles (Figure 4 G-I: co-localization with the axonal marker-NFM), as well as in enteric nerves (plexus of Meissner, Figure $3 \mathbf{J}$-L: co-localization with the axonal markerNFM). In peripheral tissues, GCDH was found expressed in epithelia (skin: Figure 4 
$\mathbf{J}$; intestinal epithelium: Figures $\mathbf{3} \mathbf{J}$ and $\mathbf{4} \mathbf{K}$ ), muscles, heart, bones (Figure $\mathbf{4} \mathbf{G}$ ), kidney (Figure $\mathbf{4}$ K), lungs (Figure $\mathbf{4} \mathbf{G}$ ) and liver (Figure $\mathbf{4}$ L). 
Table 1: Differential GCDH expression in adult and embryonic rat tissues: observations taken from at least three biological replicates

\begin{tabular}{|c|c|c|c|}
\hline \multicolumn{4}{|l|}{ Adult } \\
\hline \multicolumn{2}{|l|}{ CNS } & \multicolumn{2}{|l|}{ Peripheral tissues } \\
\hline Cerebral cortex & ++ & Skin (epithelial cells) & + \\
\hline Pyramidal neurons (layers III \& V) & +++ & Skeletal muscle & ++ \\
\hline Olfactory bulbs & + & Heart muscle & $-/++$ \\
\hline Striatum & ++ & Liver & +++ \\
\hline Hippocampus & ++ & Kidney & ++ \\
\hline Thalamus & + & Glomeruli & - \\
\hline Cerebellum & ++ & Proximal tubules & +++ \\
\hline Basket cells (molecular layer) & ++ & Juxtamedullary zone tubules & ++ \\
\hline Purkinje neurons & +++ & Intestine (epithelium) & +++ \\
\hline Granular neurons & + & Enterocytes & +++ \\
\hline Deep cerebellar nuclei & + & L cells (incl. axon-like plexus) & +++ \\
\hline Pons & ++ & Spleen & - \\
\hline Medulla oblongata & ++ & & \\
\hline Neurons (incl. dendrites \& axons) & + & & \\
\hline Oligodendrocytes & + & & \\
\hline Astrocytes & - & & \\
\hline
\end{tabular}

\section{Embryo}

Nervous system

Brain

Spinal cord

Dorsal root ganglia

Peripheral axons

Enteric nerves (plexus of Meissner)

Peripheral tissues

+++

$++$

$+++$

$+++$

$+++$
Skin (epithelial cells)

Muscles

Bones

Liver

Kidney

Intestine (epithelium)

Lungs

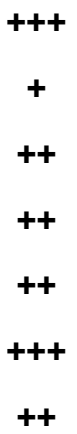

$++$ 


\section{Discussion}

Here we report the differential GCDH expression in the nervous system and peripheral organs in adult rats and during rat embryonic development at E13, E15 and $\mathrm{E} 17$.

\section{GCDH expression in adult rat brain}

GCDH expression and its function in the liver have been studied extensively, due to the major role of liver in amino acid oxidation. The high amounts of GA metabolites observed in the brain of GA-I patients (Strauss and Morton, 2003) were considered to be the consequence of an accumulation of circulating metabolites produced by liver. However, the hepatic $\mathrm{Gcdh}^{-/}$mouse model (with reduced hepatic GCDH activity) and further studies by Sauer et al., suggested an independent de novo synthesis of GA metabolites in the brain and their subsequent accumulation (trapping) in the brain as a result of a limited efflux rate through the blood-brain barrier (Sauer et al., 2006, Keyser et al., 2008). This hypothesis raised questions about GCDH function and expression in the brain. Since no transporter for the uptake of GA-I metabolites into neurons has been identified so far (Sauer et al., 2010), neuronal production of GA-I metabolites could be the main mechanism leading to brain damage in GA-I patients, who have no functional GCDH enzyme.

GCDH is expressed in neurons of all brain regions. This confirms the results of a previous study in which GCDH expression was shown in different structures of the mouse brain by western blotting and RT-PCR (Woontner et al., 2000). However, our results obtained by GCDH immunolocalization revealed a slightly divergent distribution of the enzyme in brain: while older studies performed by western blotting did not reveal any remarkable protein expression in striatal neurons (Woontner et al., 2000), we observed a predominant immune signal in cortex and cerebellar Purkinje cells, but also a homogenous expression with moderate intensity in other brain structures (including striatum). Olivera et al. showed in isolated astrocytic and neuronal cultures as well as co-cultures of neurons on astrocyte feeder layers derived from Gcdh -/- mice that Gcdh -/- astrocytes produce GA and 3OHGA after pretreatment with lysine which leads to death of striatal and cortical neurons. No deleterious effect was observed on neuronal cultures exposed to lysine (OliveraBravo et al., 2015). However, this still does not explain why striatum is the main 
affected brain area in GA-I patients undergoing an encephalopathic crisis. The particular vulnerability of striatal neurons can thus not be explained by the GCDH expression pattern.

In adult rat CNS neuronal dendrites and soma were stained prominently by GCDH immunostaining. Since GCDH is a mitochondrial enzyme, it is plausible that the observed immunostaining reflects the mitochondrial density in different brain regions or brain cells. In fact, the mitochondrial content in different cells is variable and correlates with the energy demand of the cells (Calabrese et al., 2001). In brain, most of the energy is required by neurons for propagation of action potentials and restoring post-synaptic ion fluxes after stimulation of the receptors by neurotransmitters. Only a small portion of energy is used by glial cells (Raichle and Gusnard, 2002). This suggests lower mitochondrial density in glial cells and would be compatible with the observed absence of GCDH in glial cells. In a previous study we have observed an increased vulnerability of glial cells to GA metabolites (mainly 3-OHGA) in an in vitro brain model for GA-I (Jafari et al., 2013). This observation could be explained by the absence of GCDH enzyme from glial cells.

It is well known that high numbers of mitochondria are in constant anterograde and retrograde movements along the axons (Saxton and Hollenbeck, 2012). Nevertheless, we observed only little GCDH expression in axons of adult rats. In neurons with high metabolic requirements, the transport and positioning of mitochondria is very important. Mitochondria are generated in the cell body and a constant fraction of them is transported into axons and dendrites depending on the local demand for ATP and other mitochondrial functions (Schwarz, 2013). Another fraction of mitochondria however appears anchored or stationary. The fact that we observed GCDH staining mainly in neuronal bodies and dendrites suggests that GCDH is predominantly expressed in a mitochondrial subpopulation that either resides in the cell body in proximity to free amino acid metabolism or is transported to dendrites where metabolic requirements are more demanding than in axons.

\section{GCDH expression in peripheral organs of adult rats}


We show that GCDH is ubiquitously expressed in peripheral organs of adult rats. The high expression in the liver is expected according to its central role in amino acid metabolism.

In kidney, the strong tubular expression might reflect an important, but yet unknown biological GCDH function. Similar to other organic acidurias, the enzymatic defect in GA-I causes an accumulation of intermediate metabolites (mainly 3-OHGA and GA) that are further eliminated through urine. However, in a subgroup of GA-I patients only very little or no metabolite excretion is detectable in urine. These so called "low excretor" patients show a relatively high residual GCDH enzyme activity of up to $30 \%$ (Busquets et al., 2000). It can be speculated that the semi-functional enzyme in the kidney of "low excretor" patients might have a role in degrading the metabolites that are produced in other organs and are transported via blood to the kidney. GA-I metabolites are polar structures and their transport through biological membranes has to be mediated by specialized membrane transporters. Sodium dependent dicarboxylate co-transporter 3 (NaC3) (Stellmer et al., 2007) and organic anion transporters (OATs) have been found to be specific transporters for GA and 3-OHGA in kidney and brain (Stellmer et al., 2007, Hagos et al., 2008, Muhlhausen et al., 2008, Lamp et al., 2011). OAT1 and NaC3 are localized in the basolateral membrane of kidney proximal tubular cells and translocate GA and 3-OHGA into these cells from where these metabolites are further excreted into urine by OAT4, which is expressed at the apical membrane. Here we show that GCDH is expressed in the same cells. We hypothesize that in "low excretor" patients the accumulating metabolites that are transported via the known transporters into proximal tubular cells could then further be metabolized by residual intracellular GCDH activity and thus become low or not detectable in urine. It has further been shown that GA and 3-OHGA accumulate in kidney of Gcdh-/- mice under high protein diet when going into encephalopathic crisis (Keyser et al., 2008). During crisis these mice show an increased renal expression pattern of NaC3 and OAT1 that appears to be an adaptive response to increased plasma GA and 3-OHGA levels. OAT1 transporters are then mislocalized to the apical membrane accompanied by a thinning of proximal tubular brush border membranes, which contributes to a functional tubulopathy (Thies et al., 2013). Recently, chronic renal failure has been described in adolescent and adult patients with GA-I (Kolker et al., 2015) thus suggesting that this is the final result of multiple 
injuries of proximal tubular cells during childhood. The renal disease appeared independently from the degree of neurological handicap. This indicates a distinct pathomechanism.

A strong GCDH expression was found in intestinal mucosa. A previous study suggested a significant role for intestinal tract in the excretion of 3-OHGA. It has been shown that high amounts of 3-OHGA are excreted in the intestine during an induced encephalopathic crisis in $\mathrm{Gcdh}^{-/}$mice (Keyser et al., 2008). Our findings support the hypothesis of an important GCDH function in the catabolism of free amino acids in intestinal mucosa. Moreover, we observed strong expression of GCDH in different classes of muscles including intestinal smooth muscles. Tryptophan is a precursor of serotonin, known to act as neurotransmitter in the gut. A recent study has shown that smooth muscle cells contract under the influence of serotonin in a dose-dependent manner (Tokita et al., 2015). From the administration of serotonin as an antidepressive drug we know that high serotonin levels lead to nausea, loss of appetite, anorexia, vomiting and diarrhea; symptoms comparable to what is observed in GA-I patients. Thus we hypothesize that GCDH has a key role in the regulation of the amount of serotonin in intestinal smooth muscle cells.

While western blotting did not reveal any GCDH expression in skeletal muscles in former studies (Woontner et al., 2000), we observed strong GCDH immunostaining in sarcomers. As already discussed above, this discrepancy might be explained by different technical approaches.

\section{GCDH expression during embryonic rat development}

Although GA-I usually presents in early childhood and following a metabolic decompensation, a subset of patients experience the "insidious onset" of the disease (Busquets et al., 2000). These patients present with developmental delay from early infancy and progressive dystonic cerebral palsy without any episodes of encephalopathic crisis (Jafari et al., 2011b). During embryogenesis we found an important and predominant GCDH expression in the central and peripheral nervous system already at very early stages, which suggests an important GCDH function in the development of the nervous system. The insidious onset of the disease as well as structural brain alterations such as macrocephaly, frontotemporal atrophy and 
delayed myelination observed in the pre-symptomatic period of the disease (Harting et al., 2009) could thus be a consequence of non-functional GCDH during embryonic brain development leading to neuronal damage already before birth. Although the strong neuronal expression persists into adulthood, its cellular localization changes during the development: in early embryonic stages, GCDH appeared to be localized predominantly in axons of both, central and peripheral neurons, whereas in adult brain it was mainly localized in neuronal bodies and dendrites. It is known that the mitochondrial distribution in cells is actively regulated as a biological response to different cellular conditions (Frederick and Shaw, 2007). When the axons elongates, mitochondria are actively recruited to them (Pilling et al., 2006) and accumulate near active growth cones of developing neurons (Morris and Hollenbeck, 1993). In stimulated cultured neurons, mitochondria move into and become stationary at active growth cones. When the growth cone activity lowers, mitochondria switch to retrograde movement towards the cell body (Morris and Hollenbeck, 1995). In adult brain, grown axons with less energetic needs, might have less stationary mitochondria compared to dendrites in which the mitochondrial content plays an essential role for the support of synaptic density and plasticity (Li et al., 2004).

In E13 and E15 embryos, GCDH was expressed ubiquitously and homogenously in peripheral organs, while in $\mathrm{E} 17$ embryos a significantly higher GCDH expression was observed in intestinal mucosa persisting into adulthood as discussed above. Surprisingly, skin was also found to express GCDH throughout the whole embryonic development. These epithelial tissues are in direct contact with amniotic fluid. Amniotic fluid contains free amino acids, which are absorbed through epithelial cells (Gurekian and Koski, 2005). We hypothesize that during embryonic development GCDH might have an important role in the catabolism of free amino acids absorbed from amniotic fluid in epithelial tissues.

\section{Conclusion}

In addition to its known expression in adult liver, GCDH is strongly expressed in neurons of the central and peripheral nervous system starting already at early stages of embryonic rat development. This suggests an important role for GCDH during brain development when the hepatic expression of the enzyme is substantially 
weaker. This finding is consistent with the observation of neuronal damage occurring in GA-I patients even before birth and without metabolic decompensation. Throughout embryonic development and persisting into adulthood GCDH is significantly expressed in epithelial cells (skin, intestinal mucosa). In adult rat proximal tubular cells also highly express $\mathrm{GCDH}$, which might be a potential explanation for the "low excretor" phenotype in GA-I. Its (patho-) physiological functions in intestinal mucosa, gastric nerves, skin, smooth and skeletal muscle, remain to be further elucidated. The detailed information on GCDH expression provides insights into its physiological functions in different organs and during embryonic development as well as of organ-specific pathophysiological processes that might contribute to the clinical phenotype observed in GA-I patients.

\section{Figure legends}

\section{Figure 1}

A: Specificity of the anti-GCDH antibody; to ascertain the specificity of the antiGCDH antibody used in this study, western blotting analysis of GCDH expression was performed in brain, kidney and liver of wild type and $\mathrm{Gcdh}^{-/}$mice respectively. A single band at $45 \mathrm{kD}$ was detected for $\mathrm{GCDH}$, in wild type animals only. $45 \mu \mathrm{g}$ (brain) and $15 \mu \mathrm{g}$ (kidney, liver) of total proteins were used in each lane, and the ubiquitous Mitochondrial Ribosomal Protein L12 (MRPL12) was used as loading control.

B: Semi-quantitative Gcdh expression in rat; E15, embryonic day 15 ; E18, embryonic day 18 ; B, brain; H, heart; L, liver; K, kidney; S, spleen. MRPL12 was used as a loading control.

\section{Figure 2: Expression of GCDH in the adult rat brain.}

A: cerebral cortex; co-localization (yellow) of GCDH (red) with the neuronal marker NeuN (green). B: striatum; co-localization (yellow) of GCDH (red) with the axonal marker p-NFM (green). C: olfactory bulb; co-localization (yellow) of GCDH (red) with the oligodendrocytic marker GalC (green). D-F: cerebral cortex, layer III; colocalization ( $\mathbf{F}$, yellow) of GCDH (D, red) with MAP-2 (E, green) in somas (asterisk) and dendritic trees (arrow) of pyramidal neurons of layer III; GCDH is mainly expressed in the cell body of pyramidal neurons. G-L: cerebellar cortex; G-I: colocalization (I, yellow) of GCDH (G, red) with p-NFM ( $\mathbf{H}$, green) in cerebellar axons, in 
particular axons of basket cells (arrow); note the expression of GCDH in Purkinje neurons (asterisk). J-L: co-localization (L, yellow) of GCDH ( $\mathbf{J}$, red) with MAP-2 (K, green) in somas (asterisk) and dendritic trees (arrow) of cerebellar Purkinje cells. Bars: $100 \mu \mathrm{m}$.

Figure 3: Expression of GCDH in rat adult peripheral tissues and in E17 rat embryonic intestine.

A: liver. GCDH (red). B,C: kidney; note the absence of GCDH from glomerulae (asterisk), and its strong expression in proximal tubules (arrow). D: skeletal muscle; note GCDH expression following the striations of muscle cells. E: heart muscle; note the segregated expression of $\mathrm{GCDH}$ in discrete heart muscle fibers. F-I: adult intestine; GCDH at low (F, red) and high magnification ( $\mathbf{G}$, red); co-localization (I, yellow) of $\mathrm{GCDH}$ (G, red) with the axonal marker p-NFM ( $\mathbf{H}$, green); note the high expression of GCDH in adult intestinal epithelial cells (enterocytes) as well as in axon-like plexus of $\mathrm{L}$ cells (arrow in I; p-NFM co-labeling), J-L: E17 intestine; colocalization ( $\mathbf{L}$, yellow) of GCDH ( $\mathbf{J}$, red) with the axonal marker p-NFM (K, green); note the high expression of GCDH in embryonic enteric nerves (plexus of Meissner; arrow in L; p-NFM co-labeling). Bars: $100 \mu \mathrm{m}$.

Figure 4: Expression of GCDH in E13, E15 and E17 rat embryos.

A-C: cerebral cortex at E13 (A), E15 (B) and E17 (C); note the high expression of GCDH in the subventricular zone at E13 (A, arrow). D-F: pallidum at E15; colocalization ( $\mathbf{F}$, yellow) of GCDH (D, red) with the neuronal marker MAP-2 (E, green). G-I: E15 rat embryonic back parasagittal section; co-localization (I, yellow) of GCDH (G, red) with the axonal marker p-NFM (H, green); note the high expression of GCDH in dorsal spinal cord (I, sp), spinal ganglions (I, sg) and their ventral horns (I, vh), vertebrae (I, v) and lung (I, I). J: E15 rat embryonic skin. K: E17 rat embryonic kidney and intestine. L: E17 rat embryonic liver at E17. Bars: $100 \mu \mathrm{m}$.

\section{Acknowledgments:}

We thank Marc Loup for technical assistance. 
Funding: This project was supported by the Swiss National Science Foundation, grant number 310030-153071 D. Ballhausen and Novartis Stiftung für medizinischbiologische Forschung grant Nr. 13C171 to D. Ballhausen.

\section{References}

Ballhausen D, Bonafe L, Braissant O (2010) DIFFERENTIAL EXPRESSION OF GLUTARYL-CoA DEHYDROGENASE IN ADULT RAT CNS, PERIPHERAL TISSUES AND DURING EMBRYONIC DEVELOPMENT. Journal of inherited metabolic disease 33:S41-S41.

Beauchamp MH, Boneh A, Anderson V (2009) Cognitive, behavioural and adaptive profiles of children with glutaric aciduria type I detected through newborn screening. Journal of inherited metabolic disease 32 Suppl 1:S207-213.

Busquets C, Merinero B, Christensen E, Gelpi JL, Campistol J, Pineda M, Fernandez-Alvarez E, Prats JM, Sans A, Arteaga R, Marti M, Campos J, Martinez-Pardo M, Martinez-Bermejo A, RuizFalco ML, Vaquerizo J, Orozco M, Ugarte M, Coll MJ, Ribes A (2000) Glutaryl-CoA dehydrogenase deficiency in Spain: evidence of two groups of patients, genetically, and biochemically distinct. Pediatric research 48:315-322.

Calabrese V, Scapagnini G, Giuffrida Stella AM, Bates TE, Clark JB (2001) Mitochondrial involvement in brain function and dysfunction: relevance to aging, neurodegenerative disorders and longevity. Neurochemical research 26:739-764.

Frederick RL, Shaw JM (2007) Moving mitochondria: establishing distribution of an essential organelle. Traffic 8:1668-1675.

Gurekian CN, Koski KG (2005) Amniotic fluid amino acid concentrations are modified by maternal dietary glucose, gestational age, and fetal growth in rats. The Journal of nutrition 135:22192224.

Hagos Y, Krick W, Braulke T, Muhlhausen C, Burckhardt G, Burckhardt BC (2008) Organic anion transporters OAT1 and OAT4 mediate the high affinity transport of glutarate derivatives accumulating in patients with glutaric acidurias. Pflugers Archiv : European journal of physiology 457:223-231.

Harting I, Boy N, Heringer J, Seitz A, Bendszus M, Pouwels PJ, Kolker S (2015) (1)H-MRS in glutaric aciduria type 1: impact of biochemical phenotype and age on the cerebral accumulation of neurotoxic metabolites. Journal of inherited metabolic disease 38:829-838.

Harting I, Neumaier-Probst E, Seitz A, Maier EM, Assmann B, Baric I, Troncoso M, Muhlhausen C, Zschocke J, Boy NP, Hoffmann GF, Garbade SF, Kolker S (2009) Dynamic changes of striatal and extrastriatal abnormalities in glutaric aciduria type I. Brain : a journal of neurology 132:1764-1782.

Hoffmann GF, Meier-Augenstein W, Stockler S, Surtees R, Rating D, Nyhan WL (1993) Physiology and pathophysiology of organic acids in cerebrospinal fluid. Journal of inherited metabolic disease 16:648-669.

Jafari P, Braissant O, Bonafe L, Ballhausen D (2011a) The unsolved puzzle of neuropathogenesis in glutaric aciduria type I. Mol Genet Metab 104:425-437.

Jafari P, Braissant O, Bonafe L, Ballhausen D (2011b) The unsolved puzzle of neuropathogenesis in glutaric aciduria type I. Molecular genetics and metabolism 104:425-437.

Jafari P, Braissant O, Zavadakova P, Henry H, Bonafe L, Ballhausen D (2013) Ammonium accumulation and cell death in a rat 3D brain cell model of glutaric aciduria type I. PloS one 8:e53735.

Keyser B, Glatzel M, Stellmer F, Kortmann B, Lukacs Z, Kolker S, Sauer SW, Muschol N, Herdering W, Thiem J, Goodman SI, Koeller DM, Ullrich K, Braulke T, Muhlhausen C (2008) Transport and 
distribution of 3-hydroxyglutaric acid before and during induced encephalopathic crises in a mouse model of glutaric aciduria type 1. Biochimica et biophysica acta 1782:385-390.

Koeller DM, Woontner M, Crnic LS, Kleinschmidt-DeMasters B, Stephens J, Hunt EL, Goodman SI (2002) Biochemical, pathologic and behavioral analysis of a mouse model of glutaric acidemia type I. Human molecular genetics 11:347-357.

Kolker S, Hoffmann GF, Schor DS, Feyh P, Wagner L, Jeffrey I, Pourfarzam M, Okun JG, Zschocke J, Baric I, Bain MD, Jakobs C, Chalmers RA (2003) Glutaryl-CoA dehydrogenase deficiency: region-specific analysis of organic acids and acylcarnitines in post mortem brain predicts vulnerability of the putamen. Neuropediatrics 34:253-260.

Kolker S, Koeller DM, Okun JG, Hoffmann GF (2004) Pathomechanisms of neurodegeneration in glutaryl-CoA dehydrogenase deficiency. Annals of neurology 55:7-12.

Kolker S, Valayannopoulos V, Burlina AB, Sykut-Cegielska J, Wijburg FA, Teles EL, Zeman J, Dionisi-Vici $C$, Baric I, Karall D, Arnoux JB, Avram P, Baumgartner MR, Blasco-Alonso J, Boy SP, Rasmussen $M B$, Burgard $P$, Chabrol B, Chakrapani A, Chapman K, Cortes ISE, Couce ML, de Meirleir L, Dobbelaere D, Furlan F, Gleich F, Gonzalez MJ, Gradowska W, Grunewald S, Honzik T, Horster $F$, loannou $H$, Jalan A, Haberle J, Haege $G$, Langereis $E$, de Lonlay $P$, Martinelli $D$, Matsumoto $S$, Muhlhausen C, Murphy E, de Baulny HO, Ortez C, Pedron CC, Pintos-Morell G, PenaQuintana L, Ramadza DP, Rodrigues E, Scholl-Burgi S, Sokal E, Summar ML, Thompson N, Vara R, Pinera IV, Walter JH, Williams M, Lund AM, Garcia-Cazorla A (2015) The phenotypic spectrum of organic acidurias and urea cycle disorders. Part 2: the evolving clinical phenotype. Journal of inherited metabolic disease 38:1059-1074.

Lamp J, Keyser B, Koeller DM, Ullrich K, Braulke T, Muhlhausen C (2011) Glutaric aciduria type 1 metabolites impair the succinate transport from astrocytic to neuronal cells. The Journal of biological chemistry 286:17777-17784.

Lenich AC, Goodman SI (1986a) The purification and characterization of glutaryl-coenzyme A dehydrogenase from porcine and human liver. The Journal of biological chemistry 261:40904096.

Lenich AC, Goodman SI (1986b) The purification and characterization of glutaryl-coenzyme A dehydrogenase from porcine and human liver. J Biol Chem 261:4090-4096.

Li Z, Okamoto K, Hayashi Y, Sheng M (2004) The importance of dendritic mitochondria in the morphogenesis and plasticity of spines and synapses. Cell 119:873-887.

Morris RL, Hollenbeck PJ (1993) The regulation of bidirectional mitochondrial transport is coordinated with axonal outgrowth. Journal of cell science 104 ( Pt 3):917-927.

Morris RL, Hollenbeck PJ (1995) Axonal transport of mitochondria along microtubules and F-actin in living vertebrate neurons. The Journal of cell biology 131:1315-1326.

Muhlhausen C, Burckhardt BC, Hagos Y, Burckhardt G, Keyser B, Lukacs Z, Ullrich K, Braulke T (2008) Membrane translocation of glutaric acid and its derivatives. Journal of inherited metabolic disease 31:188-193.

Olivera-Bravo S, Ribeiro CA, Isasi E, Trias E, Leipnitz G, Diaz-Amarilla P, Woontner M, Beck C, Goodman SI, Souza D, Wajner M, Barbeito L (2015) Striatal neuronal death mediated by astrocytes from the Gcdh-/- mouse model of glutaric acidemia type I. Human molecular genetics 24:4504-4515.

Pilling AD, Horiuchi D, Lively CM, Saxton WM (2006) Kinesin-1 and Dynein are the primary motors for fast transport of mitochondria in Drosophila motor axons. Molecular biology of the cell 17:2057-2068.

Raichle ME, Gusnard DA (2002) Appraising the brain's energy budget. Proceedings of the National Academy of Sciences of the United States of America 99:10237-10239.

Sauer SW, Okun JG, Fricker G, Mahringer A, Muller I, Crnic LR, Muhlhausen C, Hoffmann GF, Horster F, Goodman SI, Harding CO, Koeller DM, Kolker S (2006) Intracerebral accumulation of glutaric and 3-hydroxyglutaric acids secondary to limited flux across the blood-brain barrier constitute a biochemical risk factor for neurodegeneration in glutaryl-CoA dehydrogenase deficiency. Journal of neurochemistry 97:899-910. 
Sauer SW, Opp S, Mahringer A, Kaminski MM, Thiel C, Okun JG, Fricker G, Morath MA, Kolker S (2010) Glutaric aciduria type I and methylmalonic aciduria: simulation of cerebral import and export of accumulating neurotoxic dicarboxylic acids in in vitro models of the blood-brain barrier and the choroid plexus. Biochimica et biophysica acta 1802:552-560.

Saxton WM, Hollenbeck PJ (2012) The axonal transport of mitochondria. Journal of cell science 125:2095-2104.

Schwarz TL (2013) Mitochondrial trafficking in neurons. Cold Spring Harbor perspectives in biology 5.

Stellmer F, Keyser B, Burckhardt BC, Koepsell H, Streichert T, Glatzel M, Jabs S, Thiem J, Herdering W, Koeller DM, Goodman SI, Lukacs Z, Ullrich K, Burckhardt G, Braulke T, Muhlhausen C (2007) 3-Hydroxyglutaric acid is transported via the sodium-dependent dicarboxylate transporter NaDC3. Journal of molecular medicine 85:763-770.

Strauss KA, Morton DH (2003) Type I glutaric aciduria, part 2: a model of acute striatal necrosis. American journal of medical genetics Part $C$, Seminars in medical genetics 121C:53-70.

Thies B, Meyer-Schwesinger C, Lamp J, Schweizer M, Koeller DM, Ullrich K, Braulke T, Muhlhausen C (2013) Acute renal proximal tubule alterations during induced metabolic crises in a mouse model of glutaric aciduria type 1. Biochimica et biophysica acta 1832:1463-1472.

Tokita Y, Akiho H, Nakamura K, Ihara E, Yamamoto M (2015) Contraction of gut smooth muscle cells assessed by fluorescence imaging. Journal of pharmacological sciences 127:344-351.

Woontner M, Crnic LS, Koeller DM (2000) Analysis of the expression of murine glutaryl-CoA dehydrogenase: in vitro and in vivo studies. Molecular genetics and metabolism 69:116-122. 
GCDH

MRPL12

WT KO WT KO WT KO

E15 E18 B $\quad H \quad L \quad K \quad S$

GCDH

MRPL12

Braissant, Jafari et al, Figure 1 


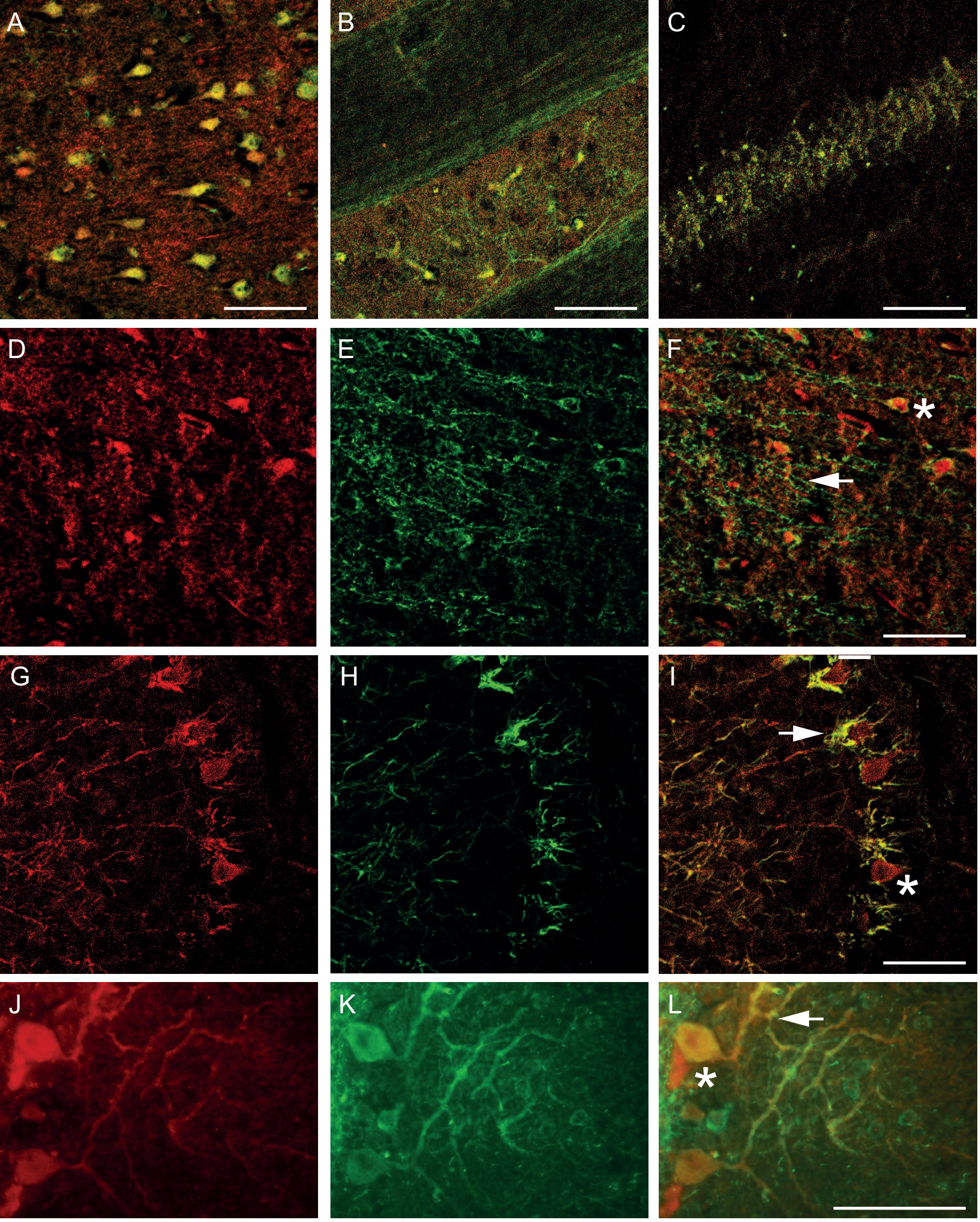

A

Braissant, Jafari et al, Figure 2
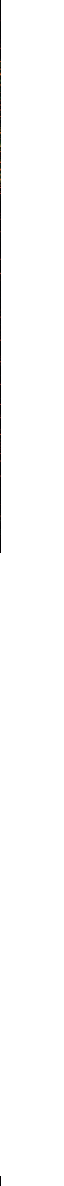


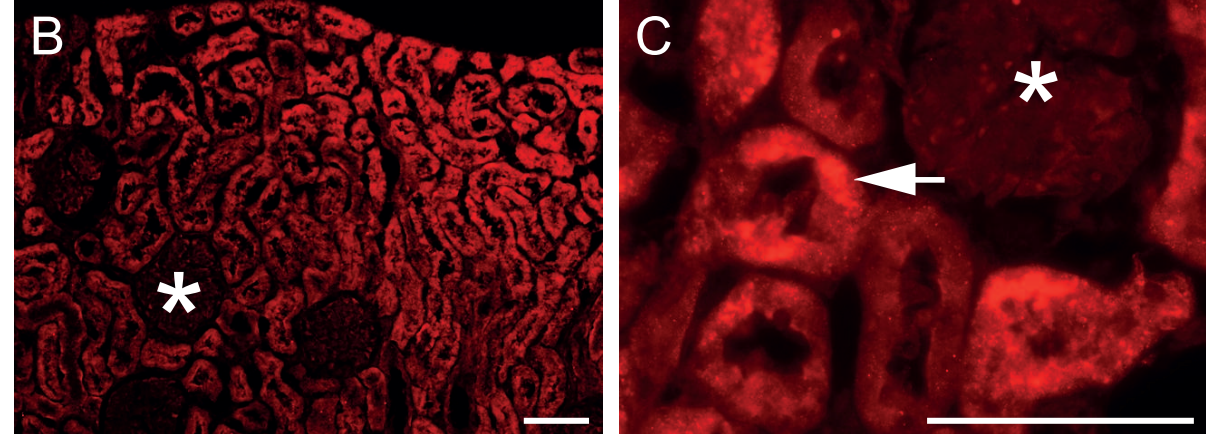

D
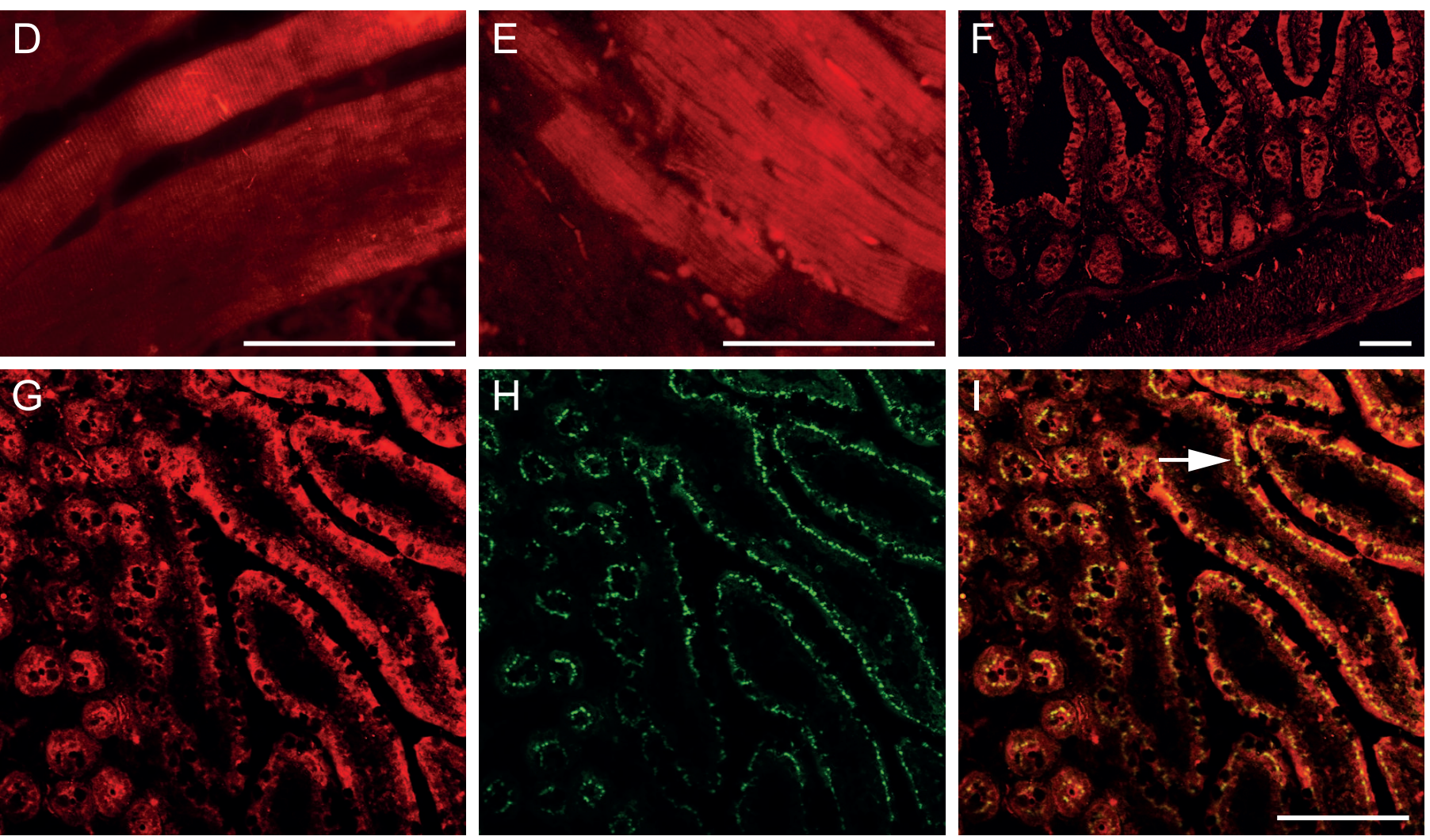

J
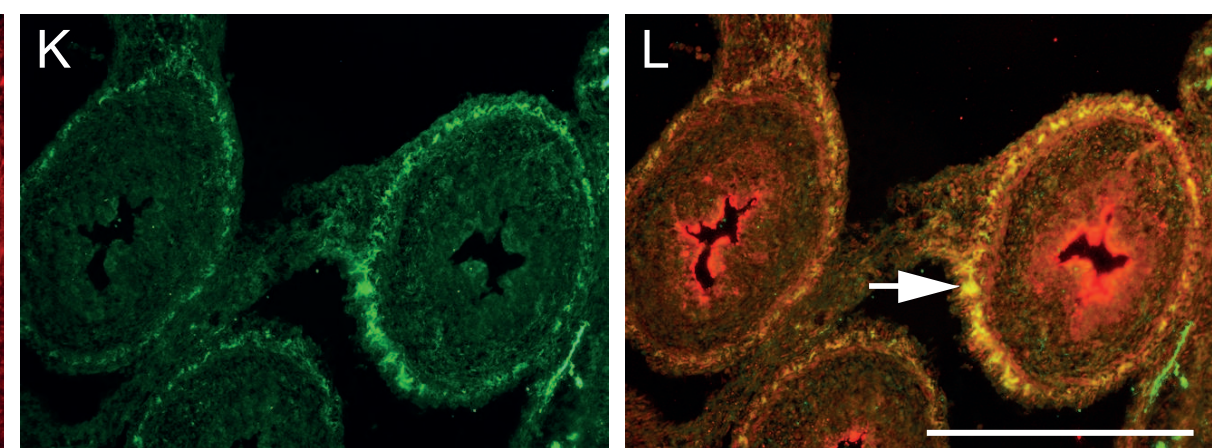

Braissant, Jafari, et al, Figure 3 
A

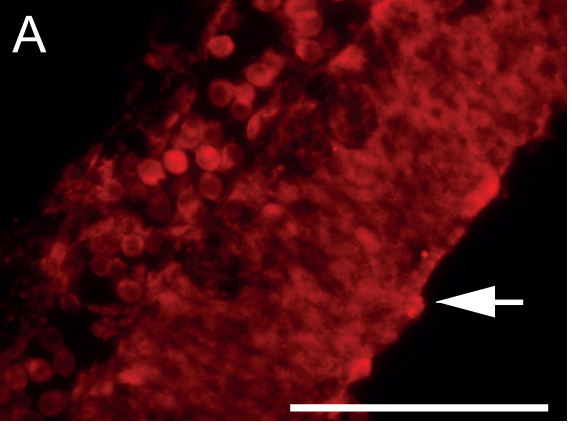

D tets

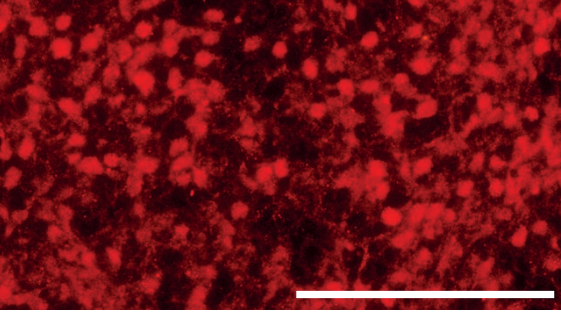

\section{G}
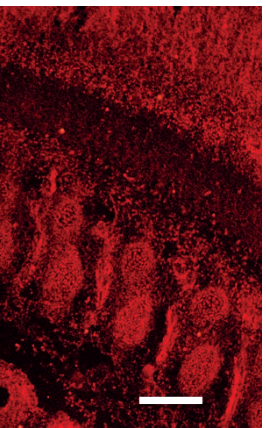

J

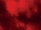
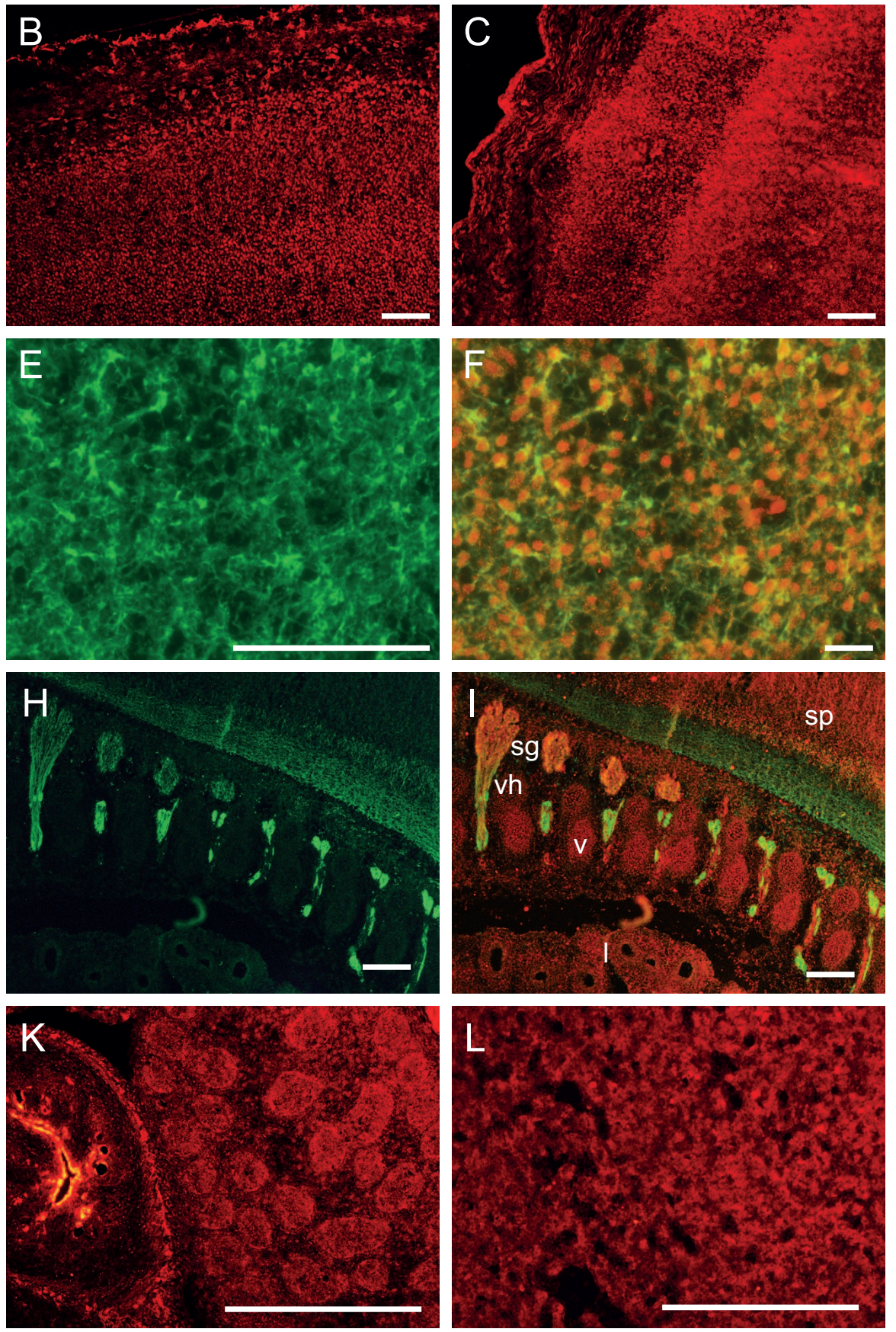

Braissant, Jafari, et al, Figure 4 